\title{
Combined use of heat-shock protein 70 and glutamine synthetase is useful in the distinction of typical hepatocellular adenoma from atypical hepatocellular neoplasms and well-differentiated hepatocellular carcinoma
}

\author{
Thuy B Nguyen ${ }^{1,2}$, Massimo Roncalli ${ }^{3,4}$, Luca Di Tommaso ${ }^{3,4}$ and Sanjay Kakar ${ }^{2}$ \\ ${ }^{1}$ Department of Pathology, University of California, San Francisco, San Francisco, CA, USA; \\ ${ }^{2}$ Department of Pathology, Mills Peninsula Medical Group, South San Francisco, CA, USA; \\ ${ }^{3}$ Department of Pathology, Humanitas Clinical and Research Center, Rozzano, Milan, Italy and ${ }^{4}$ Department \\ of Medical Biotechnology and Translational Medicine (BIOMETRA), University of Milan, Milan, Italy
}

\begin{abstract}
Well-differentiated hepatocellular carcinoma can mimic high-grade dysplastic nodule in cirrhotic liver and hepatocellular adenoma in non-cirrhotic liver. This study evaluates the efficacy of combined use of heat-shock protein 70 (HSP70), glutamine synthetase (GS) and glypican-3 in this setting. Immunohistochemistry for these three markers was done in 17 typical hepatocellular adenoma, 15 high-grade dysplastic nodules, 20 atypical hepatocellular neoplasms (14 clinically atypical and 6 pathologically atypical), 14 very well-differentiated hepatocellular carcinoma, and $\mathbf{4 3}$ well-differentiated hepatocellular carcinoma. All three markers were negative in typical adenomas. HSP70 was positive in 10,71 , and $67 \%$ of atypical neoplasms, very well-differentiated and well-differentiated HCC, respectively, while GS was positive in 60,50 , and $60 \%$ of atypical neoplasms, very well-differentiated and well-differentiated hepatocellular carcinoma, respectively. Glypican-3 was negative in all atypical neoplasms and very well-differentiated hepatocellular carcinoma, and was positive in $27 \%$ of well-differentiated hepatocellular carcinoma. Positive staining with at least one marker (HSP70 and/or GS) was seen in $85 \%$ of very well-differentiated hepatocellular carcinoma, which was similar to well-differentiated hepatocellular carcinoma $(78 \%, P=0.4)$, and pathologically atypical cases $(100 \%, P=0.5)$, but significantly higher compared with clinically atypical cases $(43 \% . P=0.03)$ and none of typical adenomas $(P<0.001)$. Positive staining with both GS and HSP70 was seen significantly more often in hepatocellular carcinoma compared with atypical neoplasms (45 vs $10 \%, P=0.004)$. Both these markers were also more often expressed in very well-differentiated hepatocellular carcinoma compared with atypical cases ( 38 vs $10 \%, P=0.06$ ). In conclusion, the combined use of GS and HSP70 can be useful in the diagnosis of very well-differentiated hepatocellular carcinoma. These stains can also help in the distinction of typical adenoma from atypical hepatocellular neoplasms. Glypican-3 has low sensitivity and is not useful in this setting.

Modern Pathology (2016) 29, 283-292; doi:10.1038/modpathol.2015.162; published online 15 January 2016
\end{abstract}

It can be challenging to differentiate well-differentiated hepatocellular carcinoma from high-grade dysplastic nodules in cirrhotic liver and hepatocellular adenoma (hepatocellular adenoma) in non-cirrhotic liver, especially when limited tissue is available for evaluation.

Correspondence: Dr S Kakar, MD, Department of Anatomic Pathology, GI-Hepatobiliary Pathology, University of California, San Francisco, 505 Parnassus Avenue, San Francisco, CA 94143, USA.

E-mail: sanjay.kakar@ucsf.edu

Received 2 September 2015; revised 11 December 2015; accepted 13 December 2015; published online 15 January 2016
The histologic features that favor hepatocellular carcinoma include wide cell plates ( $>3$ cells thick), loss of the reticulin framework, pseudoacinar architecture, small cell change, cytologic atypia, mitotic activity, stromal invasion, and vascular invasion. ${ }^{1}$ However, many of these features may be absent in well-differentiated hepatocellular carcinoma and some features such as pseudoacinar architecture, mild nuclear atypia, and focally thick cell plates can be seen in hepatocellular adenoma as well as in high-grade dysplastic nodules. ${ }^{2}$ Loss and fragmentation of reticulin network on reticulin $\operatorname{stain}^{3}$ 
and diffuse sinusoidal CD34 staining on immunohistochemistry ${ }^{4}$ can be helpful, but the overlapping findings between well-differentiated hepatocellular carcinoma and other hepatocellular lesions are not uncommon.

Heat-shock proteins are highly conserved proteins that have a critical role in protecting the cells during stress by acting as chaperones for proteins. Heat-shock protein 70 (HSP70) can block apoptosis at several levels, and is thought to have a carcinogenic role related to its antiapoptotic activity. 5,6 Antisense constructs of HSP70 have been shown to sensitize cancer cells to apoptosis and to eradicate tumors like glioma, breast carcinomas, and colon carcinomas in several models. ${ }^{7,8}$ Elevated gene expression of HSP70 has been demonstrated in hepatocellular carcinoma in several studies ${ }^{9-11}$ and is the most upregulated gene in early hepatocellular carcinoma compared with non-neoplastic liver. ${ }^{10}$ Immunohistochemical overexpression of HSP70 has been reported in hepatocellular carcinoma in several studies, ${ }^{12,13}$ and has been related to relapse as well as aggressive histologic features such as vascular invasion, high Edmondson grade, high stage, and high Ki-67 index in hepatocellular carcinoma. ${ }^{13-15}$

$\beta$-Catenin is a key component of the Wnt-signaling pathway and has an important role in cell adhesion and proliferation. Mutations in exon 3 of the $\beta$ catenin gene are seen in approximately $20 \%$ of hepatocellular carcinomas, and in up to $40 \%$ of hepatocellular carcinomas arising in the setting of hepatitis C. ${ }^{16}$ Exon 3 mutations lead to nuclear translocation of $\beta$-catenin with resultant transcriptional upregulation of glutamine synthetase (GS), an enzyme that catalyzes the synthesis of glutamine from glutamate and ammonia. In normal liver, GS is expressed in 1-3 rims of hepatocytes in the centrizonal region. ${ }^{17} \mathrm{GS}$ is one of the target genes that are overexpressed by nuclear translocation of $\beta$-catenin as a result of exon $3 \beta$-catenin mutation, and possibly by mutations in other genes involved in the Wntsignaling pathway. Diffuse expression of GS shows high concordance with the presence of $\beta$-catenin mutation in hepatocellular tumors, and is a more sensitive marker for $\beta$-catenin activation than nuclear staining with $\beta$-catenin. ${ }^{18,19}$

Diffuse GS expression has been reported in 13-70\% of early hepatocellular carcinoma and $10-15 \%$ of high-grade dysplastic nodules. GS expression in highgrade dysplastic nodules is typically focal. ${ }^{20,21}$ Diffuse GS staining in hepatocellular adenoma is often due to $\beta$-catenin activation, and many of these cases show cytologic abnormalities and frequent association with hepatocellular carcinoma. ${ }^{18,19}$ It has been suggested that most hepatocellular adenomas with diffuse GS staining may represent extremely well-differentiated variant of hepatocellular carcinoma. ${ }^{19,22}$

Glypican-3 is an oncofetal protein that is expressed in hepatocellular carcinoma but not in normal liver or hepatocellular adenoma. ${ }^{23-26}$
Glypican-3 has high sensitivity for poorlydifferentiated hepatocellular carcinoma, but the sensitivity is low $(\sim 50 \%)$ in well-differentiated hepatocellular carcinoma. ${ }^{27}$ Glypican-3 expression in high-grade dysplastic nodule has been variously reported in the literature with a range of $7-43 \% .{ }^{23,26}$

Two studies by Di Tommaso et $a l^{20,28}$ have demonstrated the utility of the combined use of HSP70, glypican-3, and GS to distinguish hepatocellular carcinoma from high-grade dysplastic nodule in biopsy and resection specimens, ${ }^{20,28}$ and these observations were corroborated by another group. ${ }^{29}$ A recent study used this combination of stains to distinguish hepatocellular carcinoma and hepatocellular adenoma, and concluded that glypican-3 and HSP70 are useful in this regard, while GS was not found to be useful. ${ }^{30}$ However, this study comprised typical cases of hepatocellular adenoma and hepatocellular carcinoma, which do not pose diagnostic problems in practice. In this study, we evaluated the utility of HSP70, glypican-3, and GS in distinguishing hepatocellular adenoma and hepatocellular carcinoma in a case cohort enriched for borderline cases and extremely well-differentiated variants of hepatocellular carcinoma.

\section{Materials and methods}

\section{Cases}

The study population comprises 107 formalin-fixed, paraffin-embedded resection cases of hepatocellular lesions obtained from the files of University of California San Francisco Medical Center. The slides were reviewed to confirm the diagnosis in all cases, which were assigned to the categories enumerated below:

(1) Clinicopathologically typical hepatocellular adenoma $(n=17)$ : tumors that occurred in women of 15-50 years of age and showed typical histologic features of hepatocellular adenoma with no morphologic atypia.

(2) Clinically atypical hepatocellular neoplasm $(n=14)$ : tumors that occurred in men (any age) or women of $>50$ years and showed typical histologic features of hepatocellular adenoma with no morphologic atypia.

(3) Pathologically atypical hepatocellular neoplasm (atypical hepatocellular neoplasm) $(n=6)$ : tumors (any age or gender) that showed histologic features largely resembling hepatocellular adenoma but with focal atypical morphological features $(<5 \%$ of the tumor) such as small cell change, pseudoacinar architecture, and thick cell plates that were insufficient for definite diagnosis of hepatocellular carcinoma.

(4) Very well-differentiated hepatocellular carcinoma $(n=13)$ : tumors with close resemblance to hepatocellular adenoma in many areas, but sufficient cytologic/architectural atypia and/or 
multifocal reticulin loss to support the diagnosis of hepatocellular carcinoma.

(5) High-grade dysplastic nodule $(n=15)$ : nodular lesions in cirrhotic liver showing cytologic/ architectural atypia (small cell change, unpaired arterioles, focally thick cell plates, and/or focal reticulin loss, no stromal invasion) that was insufficient for definite diagnosis of hepatocellular carcinoma.

(6) Well-differentiated hepatocellular carcinoma $(n=43)$ : well-differentiated tumors arising in non-cirrhotic liver $(n=20)$ or cirrhotic liver $(n=23)$ with obvious features of hepatocellular carcinoma such as thick cell plates, small cell change, stromal invasion, and/or reticulin loss in most areas of the tumor.

\section{Immunohistochemistry}

Formalin-fixed and paraffin-embedded tissue was used to perform immunohistochemistry for glypican-3, GS, and HSP70 (Table 1). Briefly, tissue sections were deparaffinized, rehydrated, and antigen retrieval was performed using heat-induced epitope retrieval in a $10 \mathrm{mmol} / \mathrm{l}$ citrate buffer, $\mathrm{pH}$ 6.0. Following peroxidase block and incubation with primary antibody for $30 \mathrm{~min}$, the sections were incubated with horseradish peroxidase-labeled secondary antibody, substrate chromagen for $10 \mathrm{~min}$, and counterstained with hematoxylin.

Table 1 Details of antibodies used for immunohistochemistry in the study

\begin{tabular}{|c|c|c|c|}
\hline Antibody & Clone & Vendor & Dilution \\
\hline Glypican-3 & $1 \mathrm{G} 12$ & BioMosaics, Burlington, VT, USA & $1: 2$ \\
\hline $\begin{array}{l}\text { Glutamine } \\
\text { synthetase }\end{array}$ & Mab302 & $\begin{array}{l}\text { Chemicon/Millipore, Billierica, } \\
\text { MA, USA }\end{array}$ & $1: 250$ \\
\hline $\begin{array}{l}\text { Heat-shock } \\
\text { protein } 70\end{array}$ & SC-24 & $\begin{array}{l}\text { Santa Cruz Biotechnology, } \\
\text { Santa Cruz, CA, USA }\end{array}$ & $1: 200$ \\
\hline
\end{tabular}

\section{Scoring}

The staining intensity was assigned a score of $0-3$ (0-absent, 1-weak, 2-moderate, and 3-strong). HSP70 was scored positive if moderate to strong nuclear staining was seen in $\geq 10 \%$ of tumor cells. Glypican-3 was considered as positive when moderate to strong nuclear, cytoplasmic and/or membranous staining was seen in $\geq 10 \%$ of tumor cells. GS was considered as diffuse positive if moderate to strong staining was observed in $\geq 50 \%$ of tumor cells. Tumors with diffuse GS staining were considered as $\beta$-Catenin activated. Two patterns of staining were observed in diffuse positive cases: (a) Diffuse homogeneous: moderate to strong staining in nearly all the tumor cells (>90\%), (b) Diffuse heterogeneous: moderate to strong staining in majority but not in all the tumor cells (50-90\%). All other GS staining patterns were scored as negative.

\section{Statistics}

The immunohistochemical expression of different markers in the individual diagnostic categories was compared using the Fisher's exact test and the Chi-square test.

\section{Results}

The clinical and pathologic characteristics of the study cases are summarized in Table 2. Subtyping of hepatocellular adenomas was not specifically done as part of this study, and the subtypes stated in the table are based on the available information. The immunohistochemical findings are summarized in Table 3. Typical hepatocellular adenomas were negative for all three markers. $\beta$-Catenin activation as evidenced by diffuse GS staining was seen in $43 \%$ of atypical hepatocellular neoplasms occurring in an

Table 2 Clinical and pathologic characteristics of study cases

\begin{tabular}{|c|c|c|c|c|c|c|c|}
\hline & $\begin{array}{l}\text { Typical hepatocellular } \\
\text { adenoma }(\mathrm{n}=17)\end{array}$ & $\begin{array}{l}\text { Clinically atypical } \\
\text { hepatocellular } \\
\text { neoplasm }(\mathrm{n}=14)\end{array}$ & $\begin{array}{c}\text { Pathologically atypical } \\
\text { hepatocellular } \\
\text { neoplasm }(\mathrm{n}=6)\end{array}$ & $\begin{array}{l}\text { Very well- } \\
\text { differentiated } \\
\text { HCC }(\mathrm{n}=13)\end{array}$ & $\begin{array}{l}\text { HCC, cirrhotic } \\
\text { liver }(\mathrm{n}=20)\end{array}$ & $\begin{array}{l}\text { HCC non-cirrhotic } \\
\text { liver }(\mathrm{n}=23)\end{array}$ & $\begin{array}{c}\text { High-grade } \\
\text { dysplastic nodule } \\
(\mathrm{n}=15)\end{array}$ \\
\hline $\begin{array}{l}\text { Oral } \\
\text { contraceptives }\end{array}$ & 5 & 0 & 0 & 2 & 0 & 1 & 0 \\
\hline $\begin{array}{l}\text { Additional } \\
\text { info }\end{array}$ & $\begin{array}{c}\text { Inflammatory: } 8 \\
\text { HNF1 } \alpha \text { inactivated: } 4 \\
\beta \text {-Catenin activated: } 0 \\
\text { Not classified: } 5\end{array}$ & $\begin{array}{c}\text { Inflammatory: } 7 \\
\text { HNF1 } \alpha \text { inactivated: } 2 \\
\beta \text {-Catenin activated: } 6 \\
\text { Not classified: } 5\end{array}$ & $\begin{array}{c}\beta \text {-Catenin activated, } \\
\text { inflammatory: } 4 \\
\beta \text {-Catenin activated, non- } \\
\text { inflammatory: } 2\end{array}$ & $\begin{array}{c}\text { Fatty liver: } 2 \\
(15 \%) \\
\text { Steatohepatitis: } \\
2(15 \%) \\
\text { Cirrhosis: none }\end{array}$ & $\begin{array}{c}\text { Hepatitis C } 5 \\
(25 \%) \\
\text { Hepatitis B } 3 \\
(15 \%) \\
\text { Steatohepatitis: } 3 \\
(15 \%) \\
\text { Biliary atresia: } 1 \\
(5 \%) \\
\text { Not known: } 8 \\
(40 \%)\end{array}$ & $\begin{array}{c}\text { All non-cirrhotic } \\
\text { liver } \\
\text { Fatty liver: } 3 \text { (13\%) } \\
\text { Steatohepatitis: } 4 \\
(17 \%)\end{array}$ & $\begin{array}{c}\text { Hepatic C: } 6 \\
\text { Hepatic B: } 3 \\
\alpha 1 \text {-antitrypsin } \\
\text { deficiency: } 1 \\
\text { Congenital hepatic } \\
\text { fibrosis: } 1 \\
\text { Not known: } 4\end{array}$ \\
\hline $\begin{array}{l}\text { Mean age } \\
\text { (years) }\end{array}$ & 39 & 45 & 46 & 48 & 52 & 48 & 50 \\
\hline $\begin{array}{l}\text { Age range } \\
\text { (years) }\end{array}$ & $23-49$ & $20-65$ & $24-57$ & $8-62$ & $10-75$ & $19-80$ & $32-62$ \\
\hline $\begin{array}{l}\text { Female } \\
\text { gender }\end{array}$ & $17(100 \%)$ & $7(50 \%)$ & $3(50 \%)$ & $1(7 \%)$ & $6(33 \%)$ & $13(56 \%)$ & $1(7 \%)$ \\
\hline
\end{tabular}


Table 3 Immunohistochemical results for glypican-3 (GPC-3), heat-shock protein 70 (HSP70), and glutamine synthetase (GS)

\begin{tabular}{|c|c|c|c|}
\hline & $G P C-3$ & HSP70 & $G S$ \\
\hline Typical hepatocellular adenoma $(n=17)$ & $\mathbf{0}$ & $\mathbf{0}$ & $\mathbf{0}$ \\
\hline Atypical hepatocellular neoplasm, all cases $(n=20)$ & $\mathbf{0}$ & $2(10)$ & $12(60)$ \\
\hline Clinically atypical $(n=14)$ & 0 & $1(7)$ & $6(43)$ \\
\hline Pathologically atypical $(n=6)$ & 0 & $1(17)$ & $6(100)$ \\
\hline HCC, all cases $(n=56)$ & $15(27)$ & $38(68)$ & $32(57)$ \\
\hline Very well-differentiated HCC $(n=13)$ & $1(7)$ & $9(71)$ & $6(50)$ \\
\hline Well-differentiated HCC, non-cirrhotic liver $(n=20)$ & $5(25)$ & $13(65)$ & $11(55)$ \\
\hline Well-differentiated HCC, cirrhotic liver $(n=23)$ & $9(39)$ & $16(70)$ & $15(65)$ \\
\hline Well-differentiated HCC, cirrhotic and non-cirrhotic liver $(n=43)$ & $14(33)$ & $29(67)$ & $26(60)$ \\
\hline High-grade dysplastic nodule $(n=15)$ & o & $1(6)$ & $1(6)$ \\
\hline
\end{tabular}

The broad diagnostic categories are in bold font, while subcategories are in font that is not bold. Figures in parenthesis reflect percentages.

Table 4 Combined immunohistochemistry using heat-shock protein 70 (HSP70) and glutamine synthetase (GS)

Both GS and HSP70

Hepatocellular adenoma, typical $(n=17)$

Atypical hepatocellular neoplasm, all cases $(n=20)$

Clinically atypical $(n=14)$

Pathologically atypical $(n=6)$

HCC, all cases $(n=56)$

Very well-differentiated HCC $(n=13)$

Well-differentiated HCC, non-cirrhotic liver $(n=20)$

Well-differentiated HCC, cirrhotic liver $(n=23)$

High-grade dysplastic nodule $(n=15)$

$\begin{aligned} & \mathbf{0} \\ \mathbf{2} & (\mathbf{1 0}) \\ 1 & (7) \\ 1 & (17) \\ \mathbf{2 5} & (\mathbf{4 5 )} \\ 5 & (38) \\ 7 & (35) \\ 13 & (57)\end{aligned}$

o
GS and/or HSP70 0

$12(60)$

$6(43)$

6 (100)

47 (84)

$11(85)$

$18(90)$

$18(78)$

2 (13)

The broad diagnostic categories are in bold font, while subcategories are in font that is not bold. Figures in parenthesis reflect percentages.

atypical clinical setting and all atypical hepatocellular neoplasms with focal atypical morphologic features. Map-like pattern of GS staining, characteristic of focal nodular hyperplasia, was not observed in any case. HSP70 was positive in one case each in both categories.

All hepatocellular carcinomas in cirrhotic and non-cirrhotic group were well-differentiated based on the WHO 2010 classification. ${ }^{31}$ Among all hepatocellular carcinomas taken together (very well-differentiated and well-differentiated), HSP70 was positive in $38(68 \%)$ cases, while GS was positive in 32 (57\%) cases. Glypican-3 was expressed in $15(27 \%)$ of hepatocellular carcinoma cases. There was no significant difference in expression of HSP70 and GS among very well-differentiated and well-differentiated hepatocellular carcinoma $(P=0.6$ and $P=0.2$, respectively). Glypican-3 staining tended to be positive more often in well-differentiated hepatocellular carcinomas (both cirrhotic and noncirrhotic) compared with very well-differentiated hepatocellular carcinoma (33 vs 7\%, $P=0.07$ ). There was no significant difference in staining for any of the three markers in cirrhotic vs non-cirrhotic liver $(P>0.1$ for all). All high-grade dysplastic nodules were negative for glypican-3; positive GS and HSP70 staining in one case each, while none of the cases were positive for both markers.

When combination of HSP70 and GS was examined, $43 \%$ of atypical hepatocellular neoplasms based on atypical clinical setting and all atypical hepatocellular neoplasms based on morphology were positive for at least one marker, while both markers were positive in one case each (Table 4) (Figure 1). Positive staining with at least one of the two markers (HSP70 and/or GS) was seen in a majority of very well-differentiated hepatocellular carcinomas (11/13, 85\%) (Figures 2 and 3), which was similar to well-differentiated hepatocellular carcinomas in non-cirrhotic liver $(18 / 20,90 \%$, $P=0.4)$ (Figure 4), cirrhotic liver $(18 / 23,78 \%$, $P=0.5)$, and atypical hepatocellular neoplasms based on morphology $(6 / 6,100 \%, P=0.5)$, but significantly higher compared with atypical hepatocellular neoplasms based on clinical setting (6/14, $43 \%, P=0.03$ ) (Table 4). Positive staining with both GS and HSP70 was seen significantly more often in hepatocellular carcinoma (all cases) compared with all cases of atypical hepatocellular neoplasms (45 vs $10 \%, P=0.004)$. Both these markers were also more often expressed in very well-differentiated hepatocellular carcinoma compared with atypical hepatocellular neoplasms with borderline statistical significance (38 vs 10\%, $P=0.06$ ).

\section{Discussion}

The distinction of hepatocellular adenoma and hepatocellular carcinoma can be challenging on 

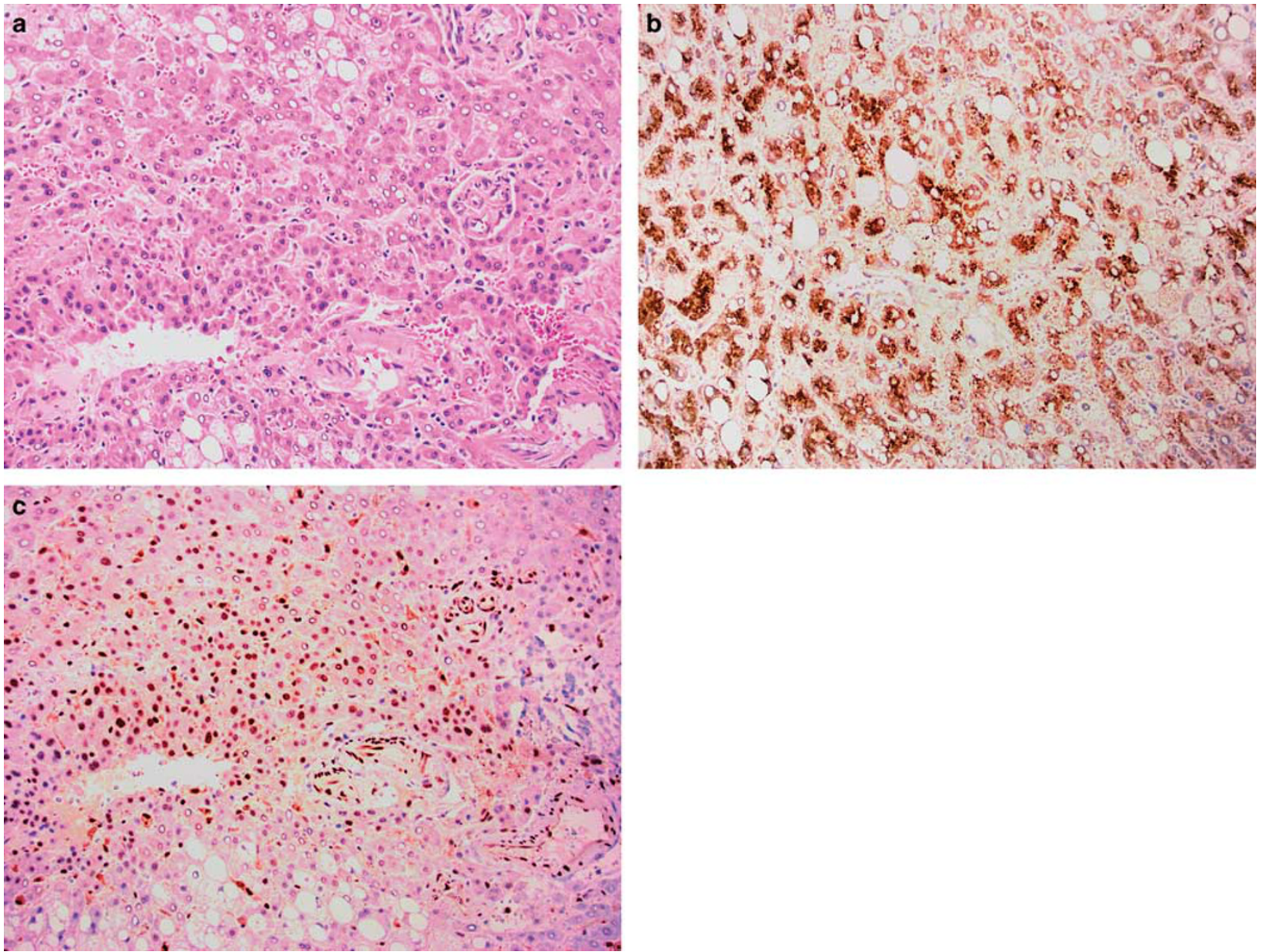

Figure 1 Atypical hepatocellular neoplasm in a 57-year old woman showing areas with small cell change and focally wide plates (a, H\&E, $\times 200$ ). Immunohistochemistry for glutamine synthetase shows diffuse heterogeneous staining (b, $\times 200)$, and HSP70 shows nuclear staining $(\mathbf{c}, \times 200)$.

needle biopsies. In some instances, tumors resembling hepatocellular adenoma that occur in men, older women or have focal atypical features may represent extremely well-differentiated variants of hepatocellular carcinoma. $^{2,19,22}$ The utility of HSP70, GS, and glypican-3 has been explored for distinction of dysplastic nodule and hepatocellular carcinoma. ${ }^{20,28,29}$ There is limited data about the utility of these markers in the distinction of hepatocellular adenoma and hepatocellular carcinoma. ${ }^{30}$

In our series, HSP70 was expressed in $68 \%$ of hepatocellular carcinomas. Similar results in hepatocellular carcinomas ranging from 46 to $72 \%$ have been reported in other studies. ${ }^{12,15,20,28-30,32}$ The very well-differentiated variant of hepatocellular carcinoma is most likely to be confused with hepatocellular adenoma. Since a majority of very well-differentiated hepatocellular carcinomas (71\%) were HSP70 positive compared with none of the typical hepatocellular adenoma, HSP70 immunohistochemistry can be useful in this differential diagnosis. Two atypical hepatocellular neoplasms
$(10 \%)$ also showed positive staining. Although outcome data are not available in this study, this suggests that HSP70 staining may prove useful in identifying cases with borderline features between hepatocellular adenoma and hepatocellular carcinoma.

Diffuse GS staining is considered to be a manifestation of $\beta$-catenin activation, and is commonly used for the identification of high-risk hepatocellular adenoma and hepatocellular carcinoma. ${ }^{18}$ In most cases, this is due to mutation in the exon 3 of the $\beta$-catenin gene. ${ }^{18,33}$ Our study shows that diffuse GS was observed in majority of atypical hepatocellular neoplasms and hepatocellular carcinomas, but none of the cases of typical hepatocellular adenoma. These results as well as earlier studies indicate that the diagnosis of hepatocellular adenoma with $\beta$-catenin activation should be made with caution even though it is a recognized WHO subtype ${ }^{31}$ as most of these tumors exhibit atypical morphologic features and focal reticulin loss and a diagnosis of hepatocellular carcinoma may be possible after careful evaluation. ${ }^{22,34}$ Concurrent or subsequent 

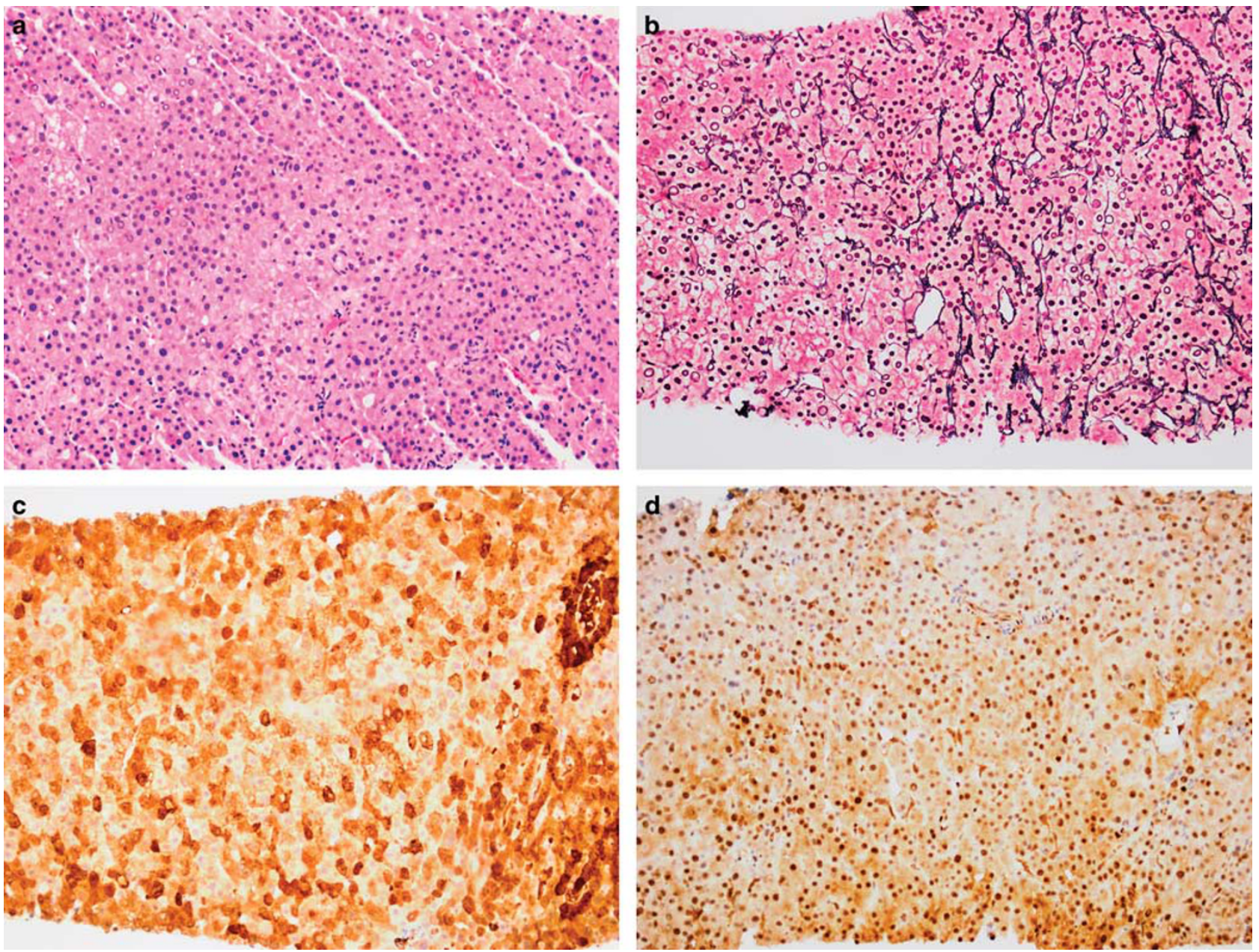

Figure 2 Very well-differentiated hepatocellular carcinoma in a 37-year old woman with small cell change and focally thick cell plates (a, $\mathrm{H} \& \mathrm{E}, \times 200)$ and fragmentation of reticulin network $(\mathbf{b}, \times 200)$. Immunohistochemistry for glutamine synthetase shows diffuse heterogeneous staining $(\mathbf{c}, \times 200)$, and HSP70 shows nuclear staining $(\mathbf{d}, \times 200)$.

hepatocellular carcinoma has been reported in up to $40 \%$ of hepatocellular adenoma with $\beta$-catenin activation, ${ }^{18}$ and most of these cases exhibit cytogenetic changes similar to hepatocellular carcinoma, ${ }^{19}$ further emphasizing that most of the $\beta$-catenin activated hepatocellular neoplasms are likely to be hepatocellular carcinoma. Hence diffuse GS staining can help in the diagnosis of hepatocellular carcinoma and in recognizing the potential high-risk outcome in atypical hepatocellular neoplasms that lack diagnostic features of hepatocellular carcinoma.

Our study shows that glypican-3 has limited value in the distinction of hepatocellular adenoma and hepatocellular carcinoma. It was positive in one-third of well-differentiated hepatocellular carcinomas, but nearly all cases of very well-differentiated hepatocellular carcinoma were negative. The low sensitivity of glypican-3 in well-differentiated hepatocellular carcinoma is well recognized. ${ }^{27}$ Since typical hepatocellular adenoma, atypical hepatocellular neoplasm, and very well-differentiated hepatocellular carcinomas are negative in nearly all cases, this stain can be omitted from diagnostic use in this setting.

High-grade dysplastic nodules were not the focus of this study, but our results were similar to those reported in the literature. Glypican-3 was negative in all cases, while GS and HSP70 were positive in $<10 \%$ of cases. Combined positivity for GS and HSP70 was not observed in any high-grade dysplastic nodule. The results of glypican-3 staining in high-grade dysplastic nodules vary widely in the literature $(7-75 \%){ }^{25-27,35}$ Our results are similar to positive staining seen in $<10 \%$ of cases in recent studies. ${ }^{20,28}$ Similarly, GS and HSP70 are positive in a small minority of high-grade dysplastic nodules. ${ }^{20,28}$

The combined use of HSP70, GS, and glypican-3 has been shown to be useful in distinguishing high-grade dysplastic nodule and hepatocellular carcinoma. Expression of two of these three markers yields a specificity of $100 \%$, while the sensitivity is $50 \%$ for biopsies and $72 \%$ for resections. ${ }^{20,28}$ One of the main goals of this study was to examine the utility of this combination in hepatocellular 

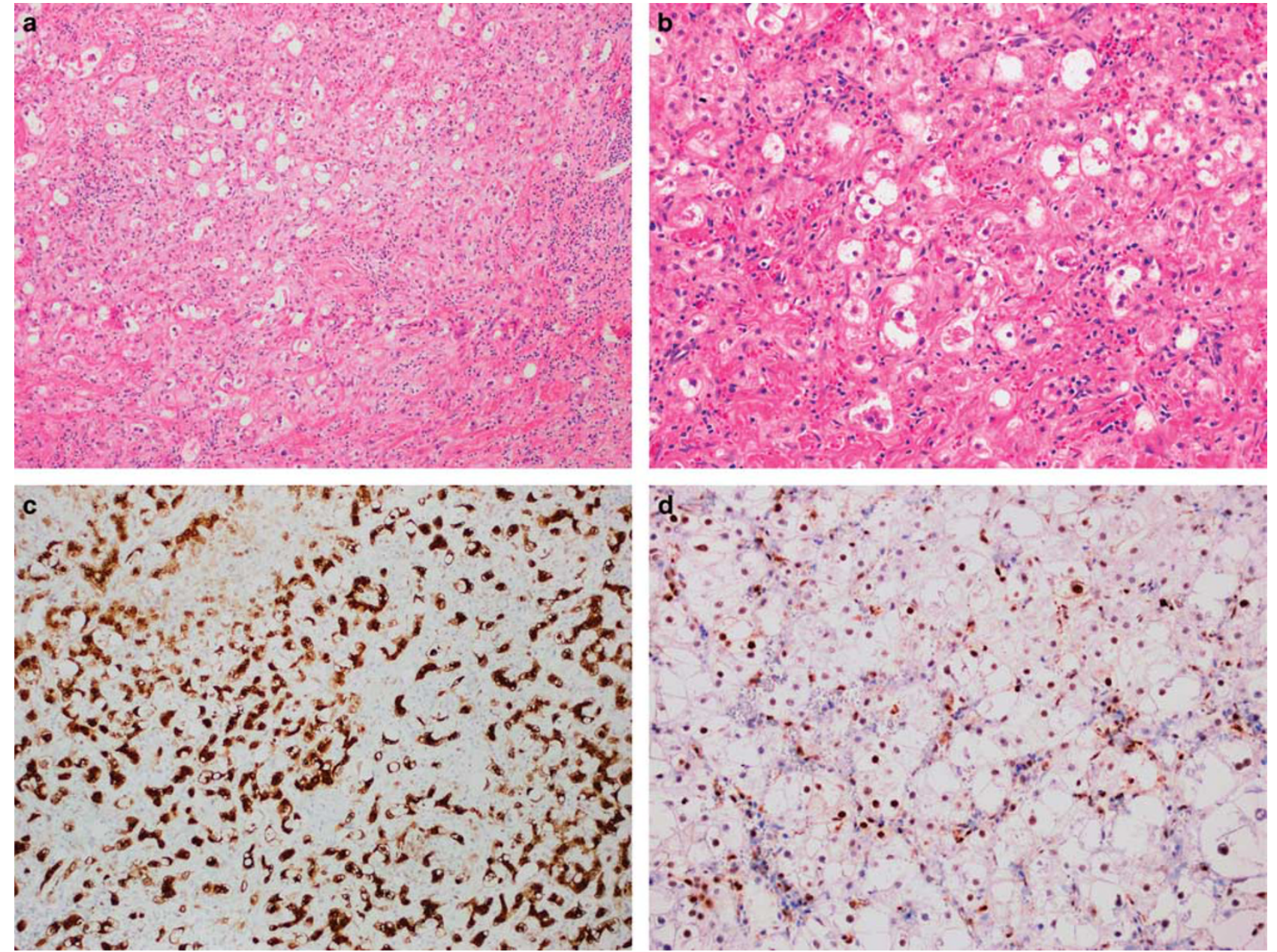

Figure 3 Very well-differentiated hepatocellular carcinoma, steatohepatitic variant, in a 52-year old woman with steatohepatitic features (a, H\&E, $\times 100, \mathbf{b}, \mathrm{H} \& \mathrm{E}, \times 200)$. Immunohistochemistry for glutamine synthetase shows diffuse heterogeneous staining $(\mathbf{c}, \times 200)$, and HSP70 shows nuclear staining $(\mathbf{d}, \times 200)$.

adenoma and hepatocellular carcinoma. Since glypican-3 was negative in nearly all these cases, its addition did not provide any additional utility.

Our results show that positive staining for both HSP70 and GS provides support for hepatocellular carcinoma even though the sensitivity is low (45\%). In contrast, both markers were positive in $10 \%$ of atypical hepatocellular neoplasms and none of the typical hepatocellular adenoma cases. Positive staining with both markers was observed in more than a third of very well-differentiated hepatocellular carcinoma, and can help in supporting the diagnosis in these challenging cases. For atypical hepatocellular neoplasms, at least one marker (GS or HSP70) was positive in $60 \%$ of cases compared with none in typical hepatocellular adenoma, indicating that positive staining with either marker in a tumor that resembles hepatocellular adenoma should raise the flag about potential for high-risk behavior. When atypical hepatocellular neoplasms with focal atypical features were considered, one of the two markers (GS or HSP70) was positive in all cases, further emphasizing the utility of combined use of GS and HSP70 in identification of atypical cases. The relatively small sample size is a limitation of our study, and further experience with a larger number of cases will help establish the true sensitivity of these markers for the diagnosis of atypical cases.

The role of these three markers for the differential diagnosis of hepatocellular adenoma and hepatocellular carcinoma was explored in a previous study, but it appears that the chosen cases did not pose diagnostic problems and borderline cases were not included. ${ }^{30}$ Our study includes both typical and atypical borderline cases, and has demonstrated the utility of these stains in a subset of borderline atypical cases, which are likely to pose a challenge in diagnostic practice. GS was not found to be useful in the study by Lagana et al. ${ }^{30}$ There are several limitations in their study that cast doubt on this conclusion. The study used $2 \mathrm{~mm}$ tissue microarray cores for immunohistochemistry. GS can show a diverse array of staining patterns in hepatocellular adenoma, and the results cannot be expressed in 

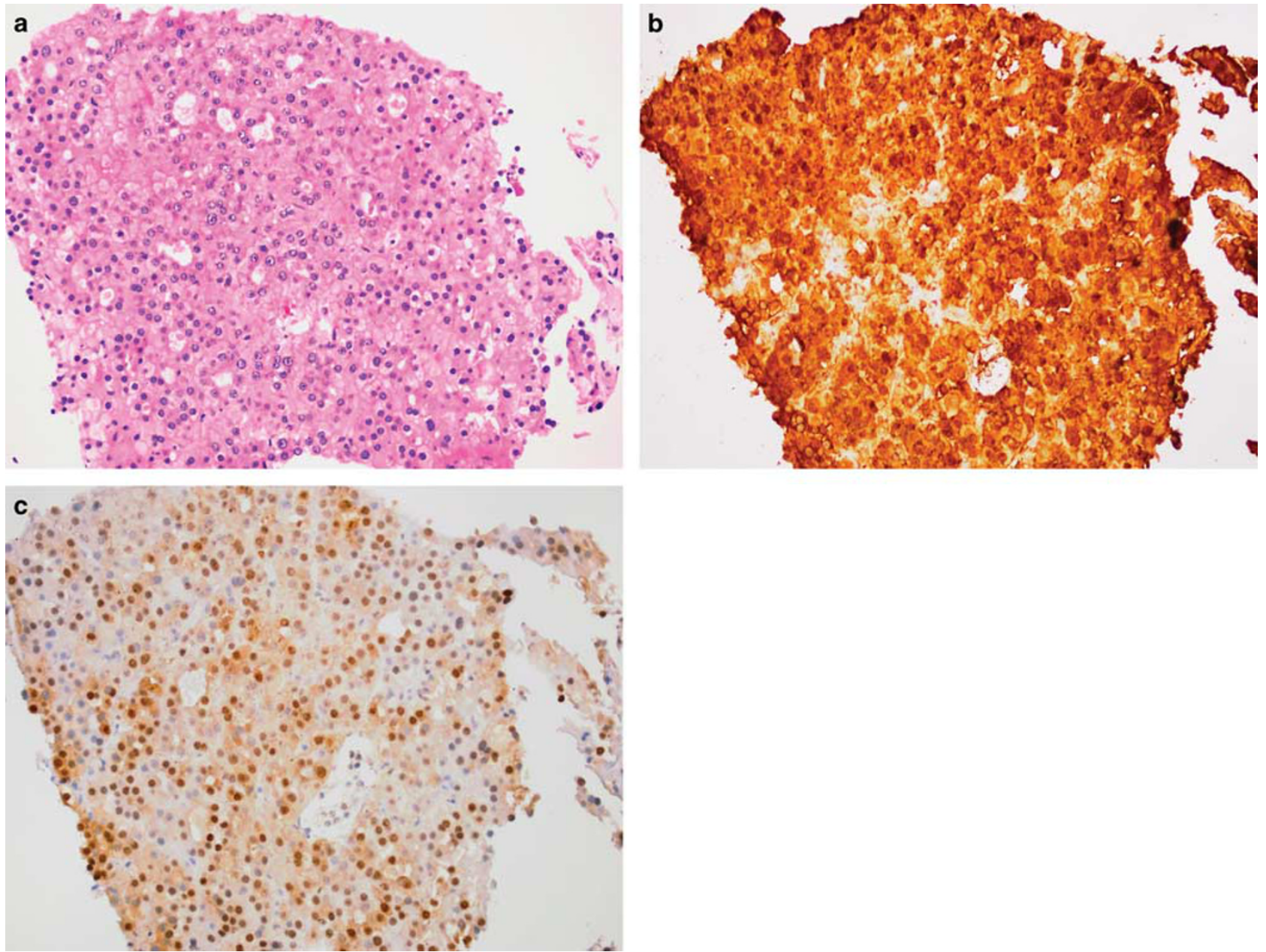

Figure 4 Well-differentiated hepatocellular carcinoma in a 65-year-old woman arising in non-cirrhotic liver (a, H\&E, 200 $\times$ ). Immunohistochemistry for glutamine synthetase shows diffuse homogeneous staining $(\mathbf{b}, 200 \times)$, and HSP70 shows nuclear staining (c, $200 \times)$.

simple negative or positive terms. Activation of $\beta$-catenin can be associated with diffuse homogeneous or diffuse heterogeneous patterns of GS staining. ${ }^{36,37}$ Although the former is easy to interpret, the latter pattern is difficult to reliable evaluate in $2 \mathrm{~mm}$ cores. Of the nine hepatocellular adenomas that were scored as GS positive in their study, three were male and some (exact number not specified) were women of $>50$ years. It has been shown that adenoma-like tumors with diffuse GS staining (signifying $\beta$-catenin activation) may represent an extremely well-differentiated hepatocellular carcinoma as reflected by cytogenetic changes resembling hepatocellular carcinoma in more than half of the cases. ${ }^{19,34}$ These tumors often occur in men. It has been argued that careful examination of morphology and reticulin staining in these cases will enable diagnosis of hepatocellular carcinoma in most instances. ${ }^{34}$ Hence, it is possible that at least some of the cases classified by Lagana et $a l^{30}$ as hepatocellular adenoma with diffuse GS represent extremely well-differentiated hepatocellular carcinoma.
In summary, immunohistochemistry for GS and HSP70 is positive in majority of very well-differentiated hepatocellular carcinomas and a subset of cases that are currently considered as atypical hepatocellular neoplasms. These stains can help in the distinction of typical hepatocellular adenomas from atypical neoplasms and hepatocellular carcinoma. Glypican-3 has low sensitivity and does not show any clinical utility in this setting. Future studies involving comparison of GS and HSP70 staining in paired biopsy and resection specimens as well as with patient outcome can further corroborate the utility of this combination.

\section{Disclosure/conflict of interest}

The authors declare no conflict of interest.

\section{References}

1 Ferrell LD, Crawford JM, Dhillon AP et al. Proposal for standardized criteria for the diagnosis of benign, 
borderline, and malignant hepatocellular lesions arising in chronic advanced liver disease. Am J Surg Pathol 1993;17:1113-1123.

2 Kakar S, Chen X, Ho C et al. Chromosomal abnormalities determined by comparative genomic hybridization are helpful in the diagnosis of atypical hepatocellular neoplasms. Histopathology 2009;55:197-205.

3 Singhi AD, Jain D, Wu TT et al. Reticulin loss in benign fatty liver: an important diagnostic pitfall when considering a diagnosis of hepatocellular carcinoma. Am J Surg Pathol 2012;36:710-715.

4 Coston WM, Loera S, Lau SK et al. Distinction of hepatocellular carcinoma from benign hepatic mimickers using Glypican-3 and CD34 immunohistochemistry. Am J Surg Pathol 2008;32:433-444.

5 Garrido C, Brunet M, Didelot C et al. Heat shock proteins 27 and 70: anti-apoptotic proteins with tumorigenic properties. Cell Cycle 2006;5:2592-2601.

6 Ruchalski K, Mao H, Li Z et al. Distinct hsp70 domains mediate apoptosis-inducing factor release and nuclear accumulation. J Biol Chem 2006;281:7873-7880.

7 Li CY, Lee JS, Ko YG et al. Heat shock protein 70 inhibits apoptosis downstream of cytochrome c release and upstream of caspase-3 activation. J Biol Chem 2000;275:25665-25671.

8 Goloudina AR, Demidov ON, Garrido C. Inhibition of HSP70: a challenging anti-cancer strategy. Cancer Lett 2012;325:117-124.

9 Takashima M, Kuramitsu Y, Yokoyama Y et al. Proteomic profiling of heat shock protein 70 family members as biomarkers for hepatitis C virus-related hepatocellular carcinoma. Proteomics 2003;3: 2487-2493.

10 Chuma M, Sakamoto M, Yamazaki K et al. Expression profiling in multistage hepatocarcinogenesis: identification of HSP70 as a molecular marker of early hepatocellular carcinoma. Hepatology 2003;37: 198-207.

11 Luk JM, Lam CT, Siu AF et al. Proteomic profiling of hepatocellular carcinoma in Chinese cohort reveals heat-shock proteins (Hsp27, Hsp70, GRP78) upregulation and their associated prognostic values. Proteomics 2006;6:1049-1057.

12 Yokoyama Y, Kuramitsu Y, Takashima $\mathrm{M}$ et al. Increased expression of heat shock protein-binding protein 1 and heat shock protein 70 in human hepatocellular carcinoma tissues. Mol Med Rep 2008;1: 197-201.

13 Tan GS, Lim KH, Tan HT et al. Novel proteomic biomarker panel for prediction of aggressive metastatic hepatocellular carcinoma relapse in surgically resectable patients. J Proteome Res 2014;13: 4833-4846.

14 Joo M, Chi JG, Lee H. Expressions of HSP70 and HSP27 in hepatocellular carcinoma. J Korean Med Sci 2005;20: 829-834.

15 Shin E, Ryu HS, Kim SH et al. The clinicopathological significance of heat shock protein 70 and glutamine synthetase expression in hepatocellular carcinoma. J Hepatobiliary Pancreat Sci 2011;18:544-550.

16 Huang H, Fujii H, Sankila et al. Beta-catenin mutations are frequent in human hepatocellular carcinomas associated with hepatitis $\mathrm{C}$ virus infection. Am J Pathol 1999;155:1795-1801.

17 Gebhardt R, Baldysiak-Figiel A, Krugel V et al. Hepatocellular expression of glutamine synthetase: an indicator of morphogen actions as master regulators of zonation in adult liver. Prog Histochem Cytochem 2007;41:201-266.

18 Bioulac-Sage P, Rebouissou S, Thomas C et al. Hepatocellular adenoma subtype classification using molecular markers and immunohistochemistry. Hepatology 2007;46:740-748.

19 Evason KJ, Grenert JP, Ferrell LD et al. Atypical hepatocellular adenoma-like neoplasms with $\beta$-catenin activation show cytogenetic alterations similar to welldifferentiated hepatocellular carcinomas. Hum Pathol 2013;44:750-758.

20 Di Tommaso L, Franchi G, Park YN et al. Diagnostic value of HSP70, glypican 3, and glutamine synthetase in hepatocellular nodules in cirrhosis. Hepatology 2007;45:725-734.

21 Sakamoto M, Mori T, Masugi Y et al. Candidate molecular markers for histological diagnosis of early hepatocellular carcinoma. Intervirology 2008;51:42-45.

22 Kakar S, Grenert JP, Paradis V et al. Hepatocellular carcinoma arising in adenoma: similar immunohistochemical and cytogenetic features in adenoma and hepatocellular carcinoma portions of the tumor. Mod Pathol 2014;27:1499-1509.

23 Zhu ZW, Friess H, Wang L et al. Enhanced glypican-3 expression differentiates the majority of hepatocellular carcinomas from benign hepatic disorders. Gut 2001;48:558-564.

24 Capurro M, Wanless IR, Sherman M et al. Glypican-3: a novel serum and histochemical marker for hepatocellular carcinoma. Gastroenterology 2003;125:89-97.

25 Yamauchi N, Watanabe A, Hishinuma $\mathrm{M}$ et al. The glypican 3 oncofetal protein is a promising diagnostic marker for hepatocellular carcinoma. Mod Pathol 2005;18:1591-1598.

26 Wang XY, Degos F, Dubois $S$ et al. Glypican-3 expression in hepatocellular tumors: diagnostic value for preneoplastic lesions and hepatocellular carcinomas. Hum Pathol 2006;37:1435-1441.

27 Shafizadeh N, Ferrell LD, Kakar S. Utility and limitations of glypican-3 expression for the diagnosis of hepatocellular carcinoma at both ends of the differentiation spectrum. Mod Pathol 2008;21:1011-1018.

28 Di Tommaso L, Destro A, Seok JY et al. The application of markers (HSP70 GPC3 and GS) in liver biopsies is useful for detection of hepatocellular carcinoma. J Hepatol 2009;50:746-754.

29 Tremosini S, Forner A, Boix L et al. Prospective validation of an immunohistochemical panel (glypican 3 , heat shock protein 70 and glutamine synthetase) in liver biopsies for diagnosis of very early hepatocellular carcinoma. Gut 2012;61:1481-1487.

30 Lagana SM, Salomao M, Bao F et al. Utility of an immunohistochemical panel consisting of glypican-3, heat-shock protein-70, and glutamine synthetase in the distinction of low-grade hepatocellular carcinoma from hepatocellular adenoma. Appl Immunohistochem Mol Morphol 2013;21:170-176.

31 Hepatocellular carcinoma, Theise N, Curado MP et al. In: Bosman FT, Carneiro F, Hruban RH, Theise ND, (eds). WHO Classification of Tumours of the Digestive System. Geneva, Switzerland: WHO Press, 2010.

32 Lim SO, Park SG, Yoo JH et al. Expression of heat shock proteins (HSP27, HSP60, HSP70, HSP90, GRP78, GRP94) in hepatitis B virus-related hepatocellular carcinomas and dysplastic nodules. World J Gastroenterol 2005;11:2072-2079. 
33 Zucman-Rossi J, Jeannot E, Nhieu JT et al. Genotypephenotype correlation in hepatocellular adenoma: new classification and relationship with HCC. Hepatology 2006;43:515-524.

34 Shafizadeh N, Genrich G, Ferrell L et al. Hepatocellular adenomas in a large community population, 2000 to 2010: reclassification per current World Health Organization classification and results of long-term follow-up. Hum Pathol 2014;45:976-983.

35 Libbrecht L, Severi T, Cassiman D et al. Glypican-3 expression distinguishes small hepatocellular carcinomas from cirrhosis, dysplastic nodules, and focal nodular hyperplasia-like nodules. Am J Surg Pathol 2006;30:1405-1411.

36 Audard V, Grimber G, Elie C et al. Cholestasis is a marker for hepatocellular carcinomas displaying beta-catenin mutations. J Pathol 2007;212: 345-352.

37 Joseph NM, Ferrell LD, Jain D et al. Diagnostic utility and limitations of glutamine synthetase and serum amyloid-associated protein immunohistochemistry in the distinction of focal nodular hyperplasia and inflammatory hepatocellular adenoma. Mod Pathol 2014;27:62-72. 